\title{
The relationship between direct predation and antipredator responses: a test with multiple predators and multiple prey
}

\author{
Scott Creel, ${ }^{1,2,6}$ Egil Dröge, ${ }^{1,2}$ Jassiel M'soka, ${ }^{1,2,3}$ DaAn Smit, ${ }^{1}$ Matt Becker,,${ }^{1,2}$ \\ Dave Christianson, ${ }^{1,4}$ and Paul Schuette ${ }^{1,5}$ \\ ${ }^{1}$ Zambian Carnivore Programme, P.O. Box 80, Mfuwe, Zambia \\ ${ }^{2}$ Department of Ecology, Montana State University, Bozeman, Montana 59717 USA \\ ${ }^{3}$ Department of National Parks and Wildlife, Department of Research, Private Bag 1, Kafue Road, Chilanga, Zambia \\ ${ }^{4}$ School of Natural Resources and the Environment, University of Arizona, Tucson, Arizona 85721 USA \\ ${ }^{5}$ Alaska Center for Conservation Science, University of Alaska Anchorage, Anchorage, Alaska 99508 USA
}

\begin{abstract}
Most species adjust their behavior to reduce the likelihood of predation. Many experiments have shown that antipredator responses carry energetic costs that can affect growth, survival, and reproduction, so that the total cost of predation depends on a trade-off between direct predation and risk effects. Despite these patterns, few field studies have examined the relationship between direct predation and the strength of antipredator responses, particularly for complete guilds of predators and prey. We used scan sampling in 344 observation periods over a four-year field study to examine behavioral responses to the immediate presence of predators for a complete antelope guild (dominated by wildebeest, zebra, and oribi) in Liuwa Plains National Park, Zambia, testing for differences in response to all large carnivores in the ecosystem (lions, spotted hyenas, cheetahs, and African wild dogs). We quantified the proportion that each prey species contributed to the kills made by each predator (516 total kills), used distance sampling on systematic line transects to determine the abundance of each prey species, and combined these data to quantify the per-capita risk of direct predation for each predator-prey pair. On average, antelopes increased their vigilance by a factor of 2.4 when predators were present. Vigilance varied strongly among prey species, but weakly in response to different predators. Increased vigilance was correlated with reduced foraging in a similar manner for all prey species. The strength of antipredator response was not detectably related to patterns of direct predation ( $n=15$ predator-prey combinations with sufficient data). This lack of correlation has implications for our understanding of the role of risk effects as part of the limiting effect of predators on prey.
\end{abstract}

Key words: antipredator behavior; predation; risk effect; vigilance.

\section{INTRODUCTION}

Most species alter their behavior in response to predation risk (Lima and Dill 1990, Tollrian and Harvell 1999). Considerable evidence shows that antipredator responses often carry costs that limit their expression when risk is low, but that the benefits of reduced predation outweigh the costs of response when risk exceeds some threshold (Skelly 1992, Tollrian and Harvell 1999, Relyea 2001b). Thus, by inducing changes in prey behavior (Winnie and Creel 2007, Periquet et al. 2012, Broekhuis et al. 2013), habitat selection (Creel et al. 2005b, Valeix et al. 2009), diet (Christianson and Creel 2010, Barnier et al. 2014), and physiology (Zanette 2003, Clinchy et al. 2004, Creel et al. 2007), the simple presence of predators can affect prey dynamics (Peckarsky et al. 1993, Boonstra et al. 1998, Christianson and Creel 2014, Cherry et al. 2016), and alter ecosystem structure and function (Schmitz et al. 1997, 2004, Peckarsky et al.

\footnotetext{
Manuscript received 31 March 2017; accepted 25 April 2017. Corresponding Editor: Liana Zanette.

${ }^{6}$ E-mail: screel@montana.edu
}

2008). In some cases, the effects of predation risk on prey dynamics are as strong, or even stronger, than the effects of direct predation itself (Peacor and Werner 2001, Werner and Peacor 2003, Preisser et al. 2005), so a good understanding of factors that affect the strength of antipredator responses is needed for sound predictions about predator-prey dynamics (Lima 1998).

Much is known about the responses of single prey species to single predators under experimental conditions, but less is known about responses to risk in more complex systems under natural conditions (Cresswell and Quinn 2013, LaManna and Martin 2016). In particular, few studies have used multi-predator, multi-prey systems to test how the magnitude of antipredator response is related to the magnitude of direct predation across species pairs. Evidence to date suggests that prey behavior is sensitive to variation in the threat posed by different predators (Cresswell and Quinn 2010, 2013), but that responses within multiple-predator-multiple-prey systems are sufficiently complex that general principles may be difficult to discern (Peckarsky and McIntosh 1998, Thaker et al. 2011). For example, Relyea (2001a) conducted an aquarium experiment in which six species of 
tadpoles were exposed to five species of caged predators. He detected antipredator responses for most predatorprey pairs, but noted that "different prey species did not respond to a particular predator the same way" and "each prey species also exhibited predator-specific responses." It is not surprising that responses differ between predator-prey pairs, but what best explains the observed variation? A prominent hypothesis is that prey should respond more strongly to predators that pose the greatest risk (Relyea 2001b). There is substantial support for this hypothesis from experimental studies, particularly with invertebrates (Kerfoot and Sih 1987, Peckarsky 1996) and fish (Werner et al. 1983), but very little is known about patterns of variation in prey response to natural variation in real predation risk in the wild, particularly among the large carnivores and ungulate prey considered in this study. Thaker et al. (2011) used spatial data from a fenced reserve to infer that ungulates avoided vegetation types that were heavily used by predators, but that "the strength of avoidance for the space use of predators generally did not correspond to the relative predation threat from those predators." In a Kenyan ecosystem, five species of ungulates increased their vigilance, reduced foraging and altered their herd sizes in response to the presence of lions and hyenas, but the strength of these responses was not detectably correlated with direct predation rates (Creel et al. 2014).

To summarize, we know that responses to risk are sensitive to many extrinsic and intrinsic variables (Boinski et al. 2003, Hopcraft et al. 2005, Balme et al. 2007, Kauffman et al. 2007, Liley and Creel 2008, Cresswell et al. 2010, Cresswell and Quinn 2013, Creel et al. 2014), but we do not understand the fundamental relationship between responses to risk and direct predation. Empirically, our understanding of this relationship is limited because few studies have measured the responses of prey to natural variation in predation risk in the wild, and fewer still have accomplished this for complete guilds of predators and prey (Lima 2002, Creel and Christianson 2008, Valeix et al. 2009, Thaker et al. 2011, Barnier et al. 2014, LaManna and Martin 2016). Conceptually, our understanding is limited because it is logical to hypothesize that the strength of antipredator responses should be positively related to the strength of direct predation (Relyea 2001b, Thaker et al. 2011), but it is also logical to hypothesize that effective antipredator responses should reduce the rate of predation, weakening the correlation or perhaps even reversing it (Lind and Cresswell 2005, Creel and Christianson 2008). These conflicting predictions reflect an important complication in the meaning of predation "risk" that was considered by Lank and Ydenberg (2003), who noted that "the most straightforward meaning and measure of predation risk could be the observed rate or probability of mortality due directly to predators", but "a quite different meaning of predation risk is the inherent probability of becoming a prey item in any particular situation if no antipredator measures are taken." That is, the observed rate of predation incorporates the effects of antipredator responses, which are expected to partially or completely offset the positive relationship between predation rates and antipredator responses that might otherwise be expected (Relyea 2001a,b, Creel and Christianson 2008). A more complete understanding of the association between direct predation and risk effects is needed to fully understand of the limiting effect of predators on prey.

Here, we tested the relationship between direct predation and the strength of antipredator responses, using data from complete ungulate and large carnivore guilds in Zambia's Liuwa Plain National Park to (1) quantify the vigilance of each ungulate prey species when each predator species was within $450 \mathrm{~m}$ (reactive vigilance) or farther than $450 \mathrm{~m}$ (proactive vigilance), (2) test for trade-offs between vigilance and foraging for each prey species, (3) quantify patterns of predation on each prey species by each predator species, (4) quantify the abundance of each prey species so that patterns of predation could be converted to individual risk, and (5) test the relationship across predator-prey pairs between direct predation and antipredator responses.

\section{Methods}

\section{Study system}

All data come from field observations between January 2011 and January 2015 within a focal study area of $1,200 \mathrm{~km}^{2}$ in the south-central portion of Liuwa Plain National Park (LPNP, 3,660 $\mathrm{km}^{2}$ ). Data were collected in all months. The Greater Liuwa Ecosystem (GLE) is located in western Zambia and is comprised of LPNP and the Upper West Zambezi Game Management Area. The ecosystem and study area are dominated by open grassland (Fig. 1) with widely distributed pans and isolated patches of open broadleaved woodland. The GLE holds one of the largest wildebeest (Connochaetes taurinus) populations in Africa, with an estimated 46,000

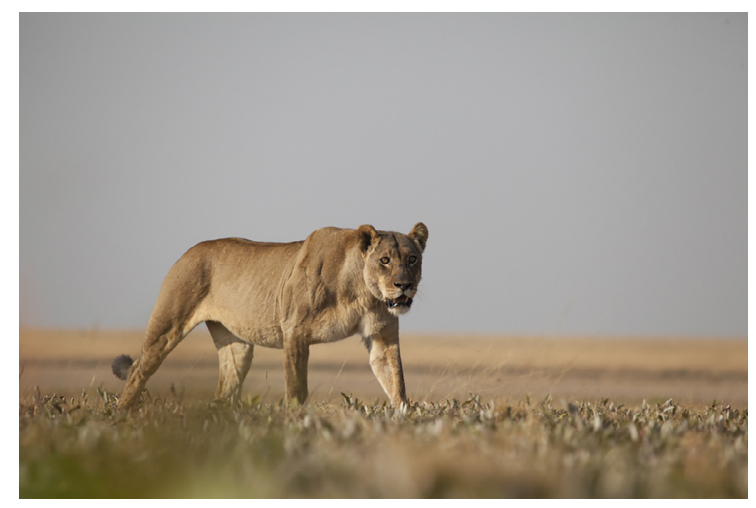

FIG. 1. The $1,200-\mathrm{km}^{2}$ study site in Liuwa Plain National Park, Zambia is highly uniform open grassland, so that variation in vegetation type is not an important component of antipredator responses as in some other systems. [Color figure can be viewed at wileyonlinelibrary.com] 
animals moving seasonally between the southern portion of LPNP in the rains and to the northwest during the dry season (M'soka et al. 2017). The ungulate guild on the focal study site is dominated by these migratory wildebeest, at local densities ranging from 6.2-60.8 individuals $/ \mathrm{km}^{2}$ ), migratory zebra (Equus quagga) at densities ranging from $1.8-8.1$ individuals $/ \mathrm{km}^{2}$, and non-migratory oribi (Ourebia ourebi) at densities ranging from 1.1-14.5 individuals $/ \mathrm{km}^{2}$. These densities were estimated with distance sampling models fit to data from a systematic transect grid that was surveyed two to three times in each year of the study, with a total transect length of 1,280 km (M'soka et al. 2017). We fit distance sampling models with the unmarked package (Chandler 2014) in R ( R Development Core Team 2008) with species-specific detection probabilities estimated from the distribution of detection distances. We focus our analysis on wildebeest, zebra, and oribi because they constitute more than $90 \%$ of the LPNP ungulate guild and more than $90 \%$ of kills made by the LPNP large carnivore guild. Less common species include red lechwe (Kobus leche leche), steenbok (Raphicerus campestris), tsessebe (Damaliscus lunatus lunatus), reedbuck (Redunca arundium), common duiker (Sylvicapra grimmia), and eland (Tragelaphus oryx). The site also holds large numbers of scrub hare (Lepus saxatilis).

The LPNP large carnivore guild includes spotted hyenas (Crocuta crocuta), lions (Panthera leo), cheetahs (Acinonyx jubatus) and African wild dogs (Lycaon pictus). Leopards (Panthera pardus) are absent. Spotted hyenas greatly outnumbered other carnivores within the study area, with 151 known hyenas in four clans, six lions in one pride, 22 wild dogs in two packs, and 17 cheetahs during the period of study (M'soka et al. 2016). To facilitate observation, we radio collared two or more individuals in each of the hyena, lion, and wild dog groups on the study site, and seven of the cheetahs (Droge et al. 2017). This carnivore guild includes two stalkers (lion and cheetah, which typically stalks despite its capacity for short pursuits) and two coursers (wild dog and hyena), allowing a direct examination of the hypothesis that hunting mode affects prey responses in a predictable manner (Schmitz 2008, Thaker et al. 2011).

\section{Behavioral responses to predator presence}

We used 344 scan-sample observation periods of 30-60 min to record herd size, composition, and the proportion of adults that were vigilant, foraging, moving, and running (a subset of moving). For each herd that we observed, we recorded the distance to the nearest known predator, predator species, predator group size, and composition, and the presence/absence of a kill at the location of the predator. To efficiently obtain these data, we first located carnivores by radio-tracking, recorded their location using a GPS, and then searched for ungulate herds. We restricted observations to herds that were not being stalked or chased, and selected ungulate herds for observation to stratify the data over distances from $50 \mathrm{~m}$ to $2 \mathrm{~km}$. We measured the straight-line distance between ungulate herds and predators using a GPS fix for each location. We did not collect data at distances $>2 \mathrm{~km}$ because prior research suggested that other, undetected predators can affect inferences for distances $>2 \mathrm{~km}$ (Creel et al. 2014). We initially tested whether anti-predator behavior was better explained by the distance to predators (treated as a continuous variable) or by a dichotomy between predators being "present" at any distance below a threshold, and "absent" beyond the threshold: comparing univariate logit-linear models of vigilance, we found that a threshold of $450 \mathrm{~m}$ revealed responses to predator presence most clearly, similar to prior research on African ungulates (Creel et al. 2014). This dichotomy distinguishes between "reactive" vigilance to monitor an immediate threat and "proactive" vigilance to monitor more diffuse risk (Courbin et al. 2015). It is possible that on some occasions a predator that we failed to detect was closer to the observed prey herd than the closest predator that we did detect, causing observations to be classified as "predator $>450 \mathrm{~m}$ " when an undetected predator was actually present within $450 \mathrm{~m}$ (false negatives). If this problem was common in our data, it would be expected to mask anti-predator responses but would not be expected to create apparent responses where none existed, and we did detect strong anti-predator responses.

We observed prey around dawn and dusk, when light conditions allowed accurate behavioral observation but the risk of predation was still high. Once a herd was selected for observation, we recorded prey species, herd size, and (for wildebeest) age-sex composition. Adults and young of the year were distinguishable for all species. A herd was operationally defined as a set of individuals with low and relatively constant inter-individual distance (typically $<10$ body lengths) that were simultaneously moving in the same speed and direction, or simultaneously stationary. We recorded behavior by scan sampling at 5-min intervals, using binoculars to scan the herd from one edge to another and a digital recorder to record the age-class, sex, and behavioral state of each individual. We recorded an animal as vigilant if it was stationary with its head held above shoulder height with the eyes and ears simultaneously focused, not chewing or ruminating. For ungulates, less-restrictive definitions of vigilance allow the possibility that an animal could simultaneously be vigilant and ruminating; we used a stringent definition of vigilance for two reasons. First, ruminating individuals are not fully vigilant, because ruminating ungulates cannot listen for predation cues, and simultaneous focus of sight and hearing is characteristic of full anti-predator vigilance in ungulates ("intense" vigilance in the sense of Periquet et al. [2012], "induced" vigilance in the sense of Blanchard and Fritz [2007]). Second, it is logical to test the foraging costs of vigilance using a definition that is sufficiently narrow to exclude active foraging and rumination (Blanchard and Fritz 2007). Less complete vigilance ("routine" vigilance in the sense of Blanchard and Fritz 
[2007]) may be compatible with rumination, but data from other ungulates (e.g., bison [Bison bison] and elk [Cervus elaphus] have shown that foraging rates are reduced even by "routine" vigilance (Fortin et al. 2004a, $b$, Blanchard and Fritz 2007) and that rumination increases predation risk (Blanchard and Fritz 2007). Because the focal prey species are all grazers, we defined foraging as remaining stationary with the head down taking bites or investigating the vegetation, alternating steps with bites/ investigation, or standing relaxed (not vigilant) while ruminating. We defined moving as taking sequential, uninterrupted steps (walking or running) without foraging behavior. We defined running as a subset of moving, with any gait faster than a walk (none of the focal species have a pacing gait, as do some ungulates). We recorded other behaviors not considered here (bedded, standing [but not vigilant], and "other" [e.g., grooming, mating]) so these behaviors do not sum to one. Observers were trained by simultaneously and independently classifying individual behavior with an experienced observer to obtain $\geq 95 \%$ congruence. Vigilance, grazing, and running were all easily and consistently classified. For each observation period, we calculated the proportion of adults that were vigilant, running (the primary antipredator responses of these species) or grazing.

\section{Direct predation}

We recorded the species (and for wildebeest, the ageclass and sex) of animals killed $(n=516)$, combining data from continuous observation of radio-collared carnivores over complete hunting periods, systematic and opportunistic locations using radiotelemetry, and opportunistic observations that did not depend on radiotelemetry. We restricted the analysis here to 452 kills for which one of the four focal carnivores was identified with high confidence (kills directly observed or judged to be $<1 \mathrm{~h}$ old, with only one carnivore species present and no sign of other species having been present), and confirmed that the patterns we report were not detectably affected by observation type $(77 \%$ of kills were directly observed). Patterns of prey selection were consistent across seasons and years for all four carnivores. Because the four carnivores kill at different rates, the data were not evenly distributed by predator species ( $n=219$ for wild dogs, 100 for hyenas, 67 for lions, and 66 for cheetahs). Because the four carnivores have strong and consistent prey preferences, the data were not evenly distributed by prey species ( $n=345$ for wildebeest, 74 for oribi, 13 for zebra). We calculated a measure of individual risk for each prey species from each predator as a selection ratio, with numerator equal to the proportion of kills that a prey species formed in a predator's diet, and the denominator equal to the proportion of the ungulate guild composed of that prey species. This ratio is equal to 1 for a prey species that is killed in proportion to its abundance, $>1$ for a species that is preferentially selected, and $<1$ for a species that is under-selected.

\section{Statistical analysis}

Our analyses used linear models fit with the $\operatorname{lm}($ ) function in the base stats package of $\mathrm{R}$, testing assumptions and goodness of fit by examining plots of scale vs. location, quantile-quantile, and residuals vs. fitted values. Where the dependent variable was a proportion and thus bounded between 0 and 1 , we used linear models with logit transformation, using diagnostic plots to confirm that this approach yielded better fit than logistic generalized linear models, following procedures outlined by Zuur et al. (2009). The full model statement within the $\operatorname{lm}()$ function was

$$
\begin{aligned}
\log \left(\frac{\text { vig }}{1-\text { vig }}\right) \sim & b_{0}+b_{1} \text { presence } \times b_{2} \text { prey }+b_{3} \text { predator } \\
& +b_{4} \text { herd }+b_{5} \text { season }
\end{aligned}
$$

where vig is the proportion of adults vigilant, presence is presence or absence of predator within $450 \mathrm{~m}$, prey is prey species identity, predator is predator species identity, herd is herd size and season is season, categorized into wet and dry. We used protected tests for interactions with predator and prey identity. Thus, because the main effect of prey identity was strong, we tested the interaction between prey identity and predator presence, and because the main effect of predator identity was weak, we did not include interactions involving this term. We used analysis of variance to compare nested models (analogous to a drop-in-deviance test for models fit with maximum likelihood) to test which effects in the full model could be excluded when testing effects of predation risk on vigilance. Our focus here is on the mean strength of response across all environmental conditions for 15 species pairs with sufficient data, but this comparison of nested models confirmed that the effects we report were not altered by group size, by season, or by interactions of these terms with prey species identity (results below; note that interactions of season and group size with prey species were tested post hoc). Finally, all of our observations were conducted in open grassland (Fig. 1) and consequently were affected little by the spatial and temporal variation in vegetation structure that complicates inference in many ecosystems (M'soka et al. 2016, Droge et al. 2017).

To test for differences in the proportions that each prey species contributed to the kills made by each predator, we calculated exact binomial 95\% confidence intervals and examined their overlaps.

To examine trade-offs between vigilance and foraging (for), we fit an exponential regression that controlled for potential differences among prey species

$$
\text { for } \sim \exp \left(b_{0}+b_{1} \text { vig }+b_{2} \text { prey }\right)
$$

To examine the relationship between antipredator responses and direct predation, we used the mean for 
each predator-prey pair to fit a regression of mean vigilance on the proportion of the diet for that predator comprised of that prey species and the per-capita risk of predation for that predator-prey pair (see Direct predation). For each of these analyses, we tested the relationship using (1) proactive vigilance only (when predators were $>450 \mathrm{~m}$ ), (2) reactive vigilance only (when predators were $<450 \mathrm{~m}$ ), (3) proactive and reactive vigilance combined, and (4) the difference between proactive and reactive vigilance. These analyses were restricted to 15 predator-prey combinations with adequate data on behavioral responses ( $n \geq 19$ observation periods).

\section{RESULTS}

\section{Responses of prey vigilance to carnivore presence: broad patterns}

The proportion of individuals vigilant more than doubled when predators were present within $450 \mathrm{~m}$ (vigilance with predator present $=0.122 \pm 0.015$, absent $=$ $0.056 \pm 0.009$ [mean \pm S.E.M.], $t=4.60, P<0.0001$ ), but superimposed on this basic response of vigilance to risk, there was significant variation among prey species (Fig. 2). Overall, oribi were appreciably more vigilant (proportion vigilant $=0.15 \pm 0.02)$ than zebra $(0.08 \pm 0.02$, $t=2.60, P=0.01)$ or wildebeest $(0.07 \pm 0.01, t=2.93$, $P=0.003)$, largely because wildebeest and zebra dropped their vigilance when no predators were nearby, but oribi did not (Fig. 2). None of the predators provoked significantly stronger responses than the others $(P=0.42$ to 0.88 for all tests of carnivore identity), but we saw marginally stronger responses to the presence of lions (2.8-fold increase in vigilance) and wild dogs (2.7-fold increase), followed by hyenas (2.3-fold increase) and cheetahs (2.2-fold increase). To summarize, vigilance responded strongly to

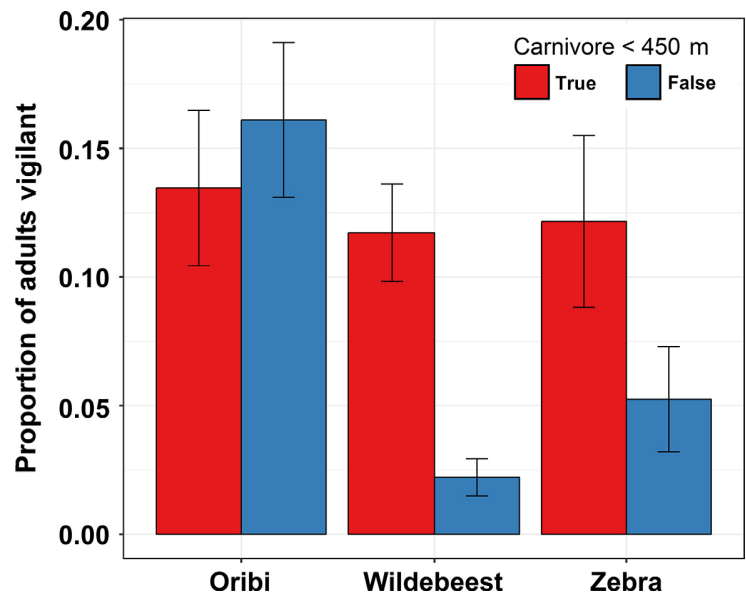

FIG. 2. When carnivores were present within $450 \mathrm{~m}$, vigilance increased by a factor of 2.44 , but there were substantial differences among prey species in the magnitude of response. Whiskers show 95\% normal approximation confidence intervals. [Color figure can be viewed at wileyonlinelibrary.com] immediate risk and varied among prey species, but differed relatively little in response to the four predator species.

Our focus here is on testing how behavioral responses to predators correlate with direct predation by those predators, when considered across all conditions over a period of four years. Although vigilance was affected by the presence of predators and varied among prey species, it was not detectably altered by variation in herd size $(b=-0.014 \pm 0.0 .001$ [mean $\pm \mathrm{SE}$ ], $t=1.41, P=0.16)$, or composition [proportion females] $b=0.004 \pm 0.006$, $t=0.68, P=0.49$ ), probably because herd sizes were relatively small and constant in this system (oribi $2.47 \pm 0.09$, zebra $13.03 \pm 2.80$, wildebeest $6.46 \pm 1.42$ ), so that prey cannot routinely rely on the strong dilution of risk that occurs in very large herds. There was no detectable interaction between the effects of herd size and prey species (all $P>0.41$ for post hoc tests). Vigilance did not vary significantly between the wet and dry seasons (effect of dry season relative to wet season $=-0.012 \pm 0.008 \mathrm{SE}, t=1.49$, $P=0.26$ ), with no detectable interaction of season with species identify (all $P>0.39$ for post-hoc tests), probably because vegetation structure does not change in a manner that appreciably affects predation risk within this study area (Fig. 1).

\section{Trade-offs between vigilance and foraging}

The proportion of individuals foraging was inversely related to the proportion vigilant (Fig. 3, $b=-0.90 \pm$ $\left.0.13, P<0.0001, r^{2}=0.16\right)$ with no detectable differences between wildebeest, oribi and zebra in the form of this trade-off $(P>0.10$ for all differences between species). As in other systems (Creel et al. 2014), the mean proportion of adults that were vigilant and the mean proportion of foraging adults summed to substantially less than one (0.64), leaving considerable scope for

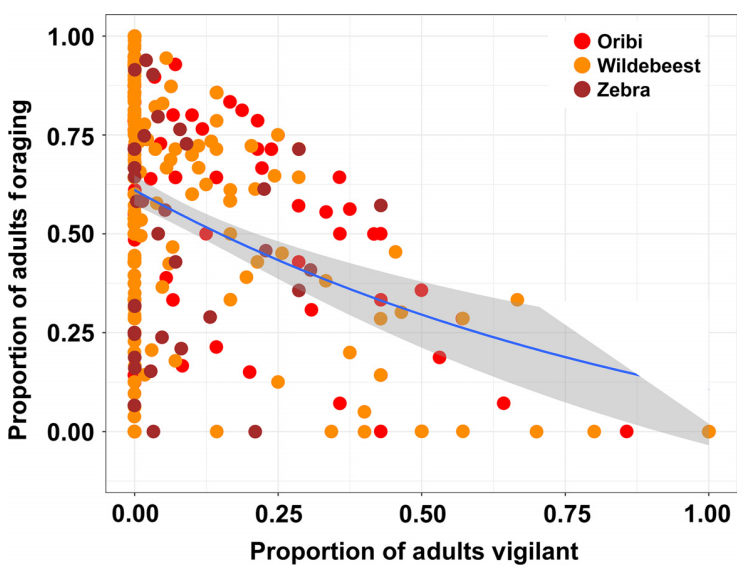

FIG. 3. Increased vigilance was associated with decreased foraging in a negative exponential manner, with no detectable differences among species in the form of this relationship. Shading shows the $95 \%$ confidence interval. [Color figure can be viewed at wileyonlinelibrary.com] 
vigilance to increase without a reduction in foraging, but we nonetheless detected an appreciable trade-off.

\section{Running in response to predators}

Running can be a direct response of ungulates to risk, though ungulates often do not run from predators that they have detected, and running was observed less than $1 / 10$ as frequently as vigilance (proportion running ranged from a minimum of 0.001 for zebra when no predator was within $450 \mathrm{~m}$ to a maximum of 0.021 for oribi when a predator was within $450 \mathrm{~m}$ ). The only detectable effects on this response were that (highly cursorial) wild dogs were more likely than the other predators to provoke flight $(t=3.512, P=0.0005)$, and wildebeest were less likely than the other prey species to run when wild dogs were present $(t=-3.25, P=0.001)$. This result aligns well with the observation that wildebeest are less likely to be killed by wild dogs if they remain stationary (Creel \& Creel 2002), a pattern that complicates the interpretation of flight as an antipredator defense when comparing several prey species.

\section{Direct predation on each prey species by each predator species}

Predation by all four carnivores was concentrated on the most common ungulates, particularly wildebeest

\section{Lion}
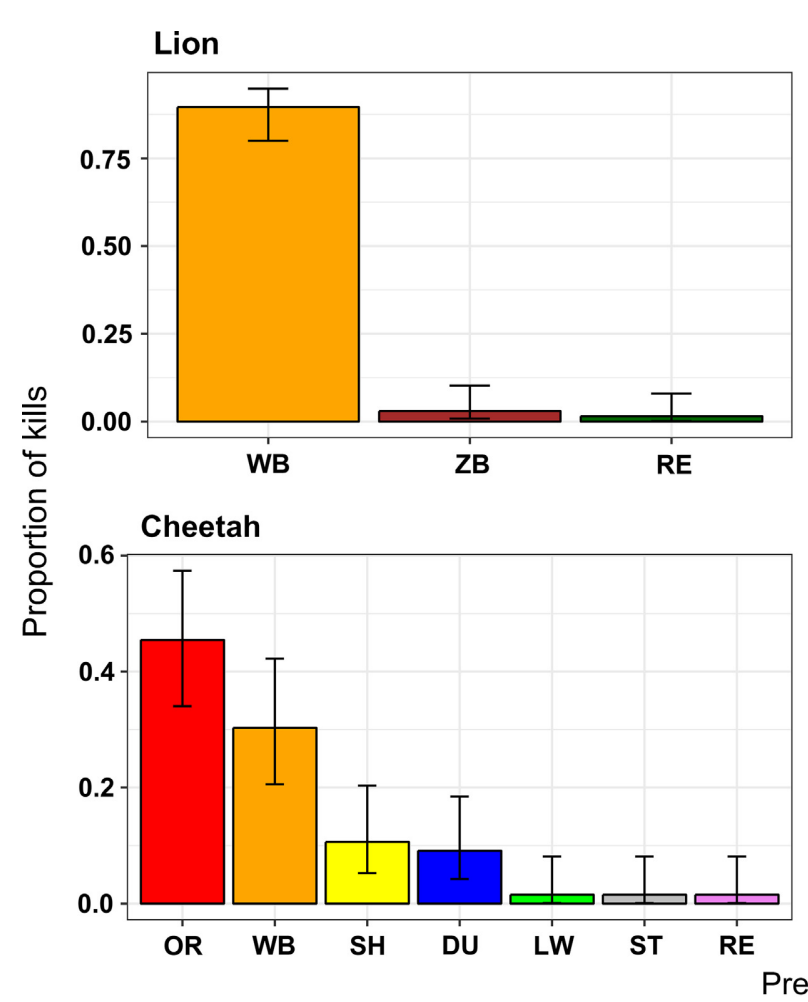

(Fig. 4). Wildebeest were the most important prey for three of the four carnivores, comprising $92 \%$ of hyena kills (95\% CI: $85-96 \%), 90 \%$ of lion kills (95\% CI: $85-96 \%), 59 \%$ of wild dog kills (95\% CI: 52-65\%), and $30 \%$ of cheetah kills (95\% CI: $20-42 \%$ ). Lions and hyenas preyed almost exclusively on wildebeest, while cheetahs and wild dogs had broader diets (Fig. 4). Cheetahs often killed oribi $(45 \%, 95 \%$ CI: $34-57 \%)$, which were also taken in appreciable numbers by wild dogs $(18 \%, 95 \% \mathrm{CI}$ : $14-24 \%$ ), but were rarely killed by hyenas and never (within our data) by lions. Together, wildebeest and oribi comprised $77 \%(95 \% \mathrm{CI}$ : $71-82 \%)$ of wild dog kills and $74 \%$ (95\% CI: $64-84 \%$ ) of cheetah kills. Scrub hares were the next most common prey for both cheetahs and wild dogs (though they are much smaller than the other species considered). Common duiker and red lechwe were rarely killed by cheetahs and wild dogs, and all other species were rarely killed by any of the predators. These patterns of prey selection were constant across seasons (and years) for all four predators, with lions and hyenas preying almost exclusively on wildebeest, and cheetahs and wild dogs consistently focusing the bulk of their predation on oribi and wildebeest under all conditions.

Because wildebeest were commonly killed by all four predators, the data were sufficient to examine patterns of predation among age-sex classes (Fig. 5). Wildebeest show rather strong sexual segregation, so that some
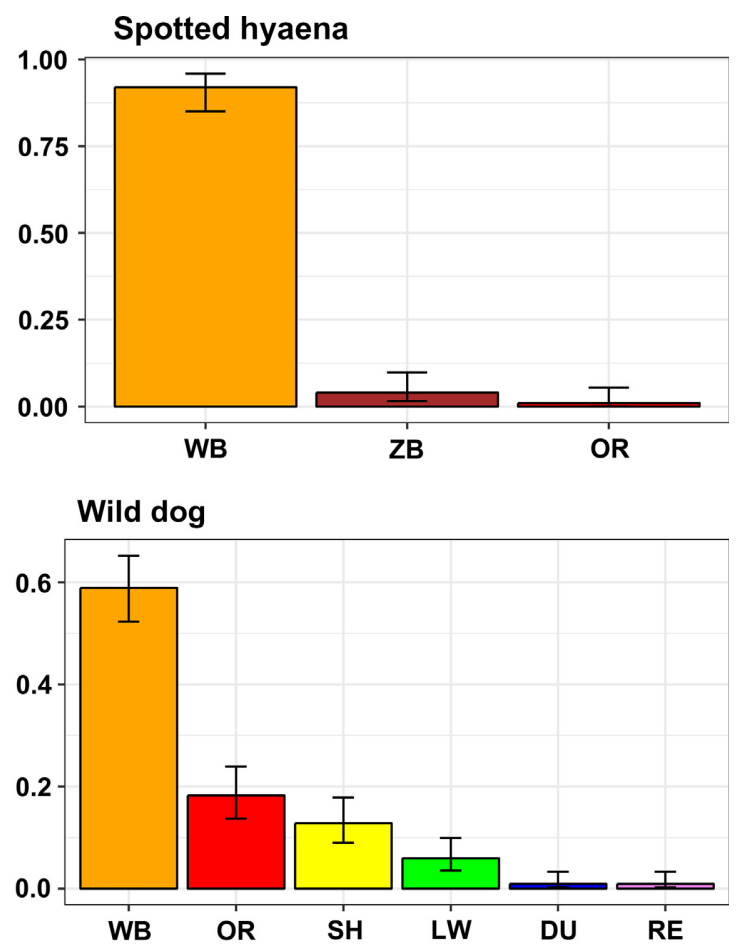

FIG. 4. The proportion of predation by each predator on each prey species varied substantially, though wildebeest were common prey for all carnivores, particularly lions and hyenas. Whiskers show binomial 95\% confidence limits. WB, wildebeest; ZB, zebra; OR, oribi; RE, reedbuck; SH, scrub hare; DU, common duiker; LW, lechwe; ST, steenbok. [Color figure can be viewed at wileyonlinelibrary.com] 
herds hold primarily females with offspring and other herds (and singletons) hold only bulls. Lions and hyenas preyed almost exclusively on wildebeest and focused heavily on adults $(86 \%$ [75-93\%] and $89 \%$ [80-94\%] respectively) mainly killing bulls, which by themselves composed half of lion and hyena kills (50\% [38-62\%] and 49\% [39-59\%], respectively). Cheetahs and wild dogs preyed less exclusively on wildebeest and focused heavily on calves $(83 \%[61-94 \%]$ and $75 \%$ [66-82\%] respectively). Thus, if antipredator responses are positively related to direct predation, then the strength of wildebeest responses should be weak for cheetahs, intermediate for wild dogs, and strong for lions and hyenas. More particularly, all-bull herds should respond strongly to lions and hyenas, but weakly to wild dogs and cheetahs.

As Fig. 6 shows, the responses of wildebeest do not support the hypothesis that antipredator responses are positively related to patterns of direct predation. Responses to cheetahs were weak (as expected), but the strongest responses were to wild dogs (contrary to expectation). The responses of wildebeest were not significantly related to predator identity, nor were they related to the magnitude of direct predation by each predator, to differences in hunting mode, or to predator group size (which was large for hyenas and wild dogs, and small for lions and cheetahs). Responses were stronger for predators that were less common.

\section{Relationships between antipredator responses, direct predation, and direct predation per-capita}

The patterns of predation on each species described thus far do not directly measure the risk of predation for individuals of each prey species, because they do not yet account for differences in prey abundance. Wildebeest were killed most often, but they were also the most common species on the study site in both the wet $\left(60.8 \pm 2.34\right.$ individuals $\left./ \mathrm{km}^{2}\right)$ and dry $(11.3 \pm 0.67$ individuals $/ \mathrm{km}^{2}$ ) seasons, followed by zebra (wet, $8.1 \pm 0.59$ individuals $/ \mathrm{km}^{2}$; dry, $2.8 \pm 0.31$ individuals/ $\mathrm{km}^{2}$ ) and oribi (wet, $2.4 \pm 0.09$; dry, $2.2 \pm 0.08$ individuals $/ \mathrm{km}^{2}$ ). In the analyses of relationships between antipredator responses and direct predation that follow, we consider both the proportion of direct predation (Figs. 4 and 5), and the proportion of direct predation relative to abundance. Some prior studies have examined the proportion of direct predation (e.g., Thaker et al. 2011) and others have considered predation relative to abundance (e.g., Creel et al. 2014): it is the second of
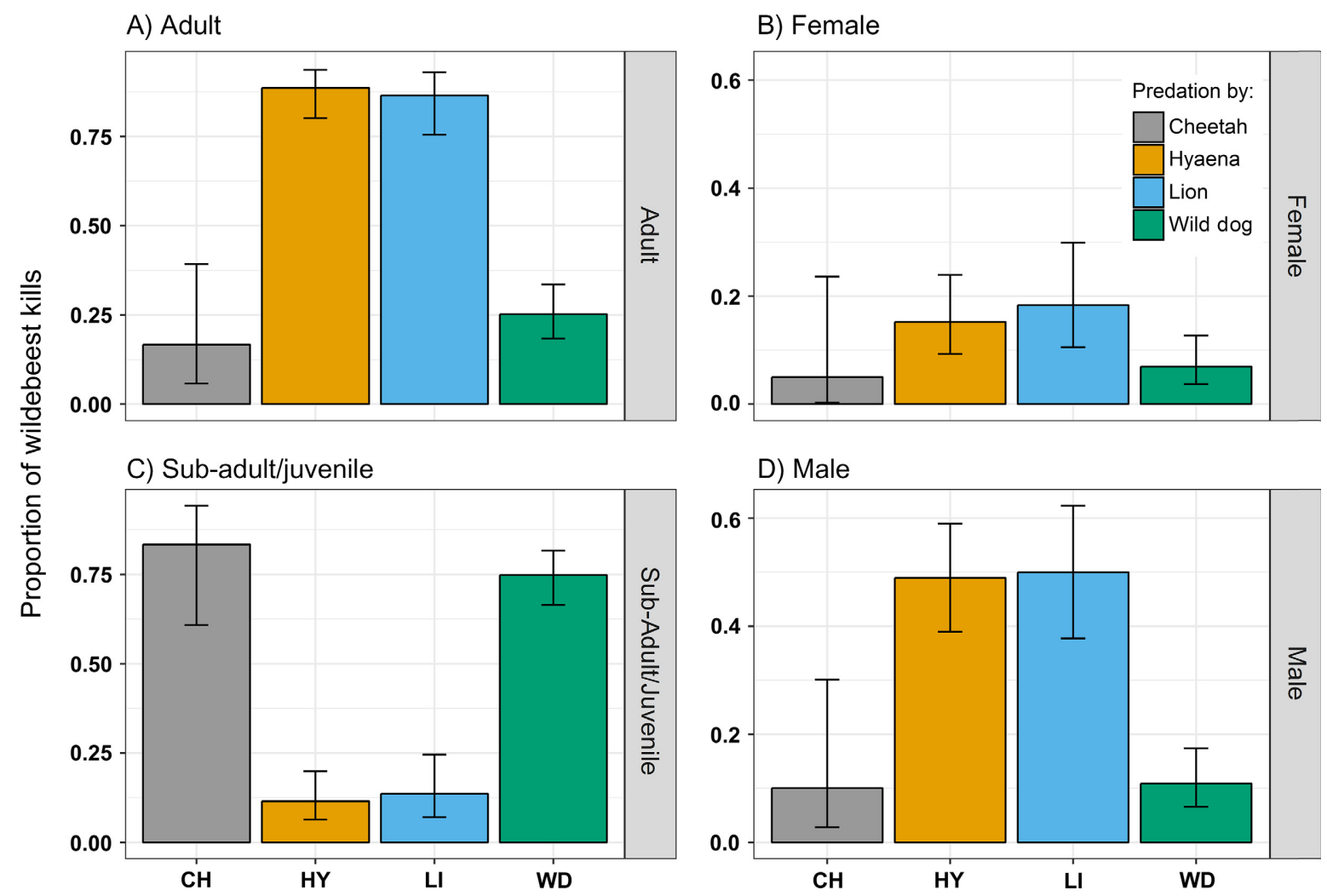

\section{D) Male}

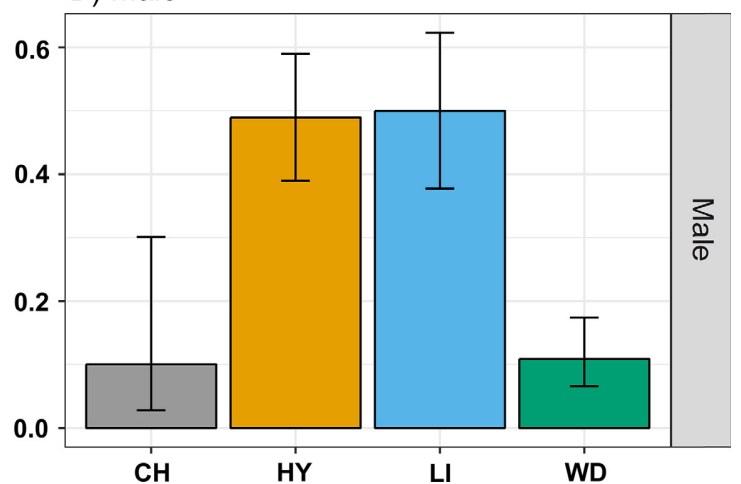

FIG. 5. (A, C) The proportion of predation by each predator on each age class of wildebeest. (B, D) The proportion of predation by each predator on male and female wildebeest adults (proportions do not sum to one because sex was not always known). Lions and hyenas killed primarily adults, particularly males, but cheetahs and wild dogs primarily killed juveniles. Whiskers show binomial 95\% confidence limits. [Color figure can be viewed at wileyonlinelibrary.com] 


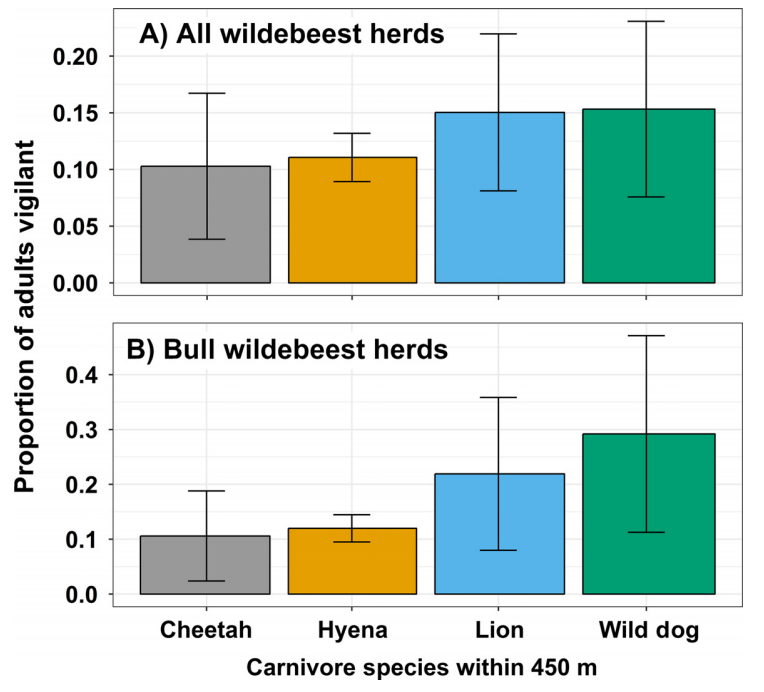

FIG. 6. (A) Vigilance levels of adult wildebeest in all herds and (B) in herds with only males in response to the presence of each carnivore species within $450 \mathrm{~m}$. Whiskers show $95 \%$ normal approximation confidence limits. [Color figure can be viewed at wileyonlinelibrary.com]

these measures that properly describes the likelihood of direct predation by each predator for individuals of each prey species.
As Fig. 7 shows, the strength of antipredator response showed no detectable relationship with direct predation $(b=-0.0093 \pm 0.054, \quad t=-0.17, \quad P=0.87, \quad n=15$ species pairs with $\geq 19$ behavioral observation periods) or with direct predation relative to abundance $(b=0.0093 \pm 0.0062, t=1.48, P=0.16, n=15$ species pairs). This lack of detectable correlation also held true when restricting the analysis to data with carnivores present within $450 \mathrm{~m}$ (i.e., reactive vigilance only), restricting the analysis to data with carnivores $>450 \mathrm{~m}$ (proactive vigilance only), and for the change in vigilance when comparing observations with carnivores $>450 \mathrm{~m}$ and observations with carnivores $<450 \mathrm{~m}$ (Fig. 7). Similarly, no correlation could be detected between running and direct predation $(b=0.0006 \pm 0.0009, t=0.67, P=0.5)$, or between running and direct predation corrected for abundance $(b=0.0004 \pm 0.0009, t=0.44, P=0.6)$.

\section{DisCUSSION}

Multispecies tests of the relationship between direct predation and the strength of antipredator responses remain rare, due to the substantial difficulty of obtaining such data, particularly for studies measuring responses to natural variation in real predation risk in the wild. For ungulates, research relating the spatial distributions of seven prey species to patterns of direct predation in

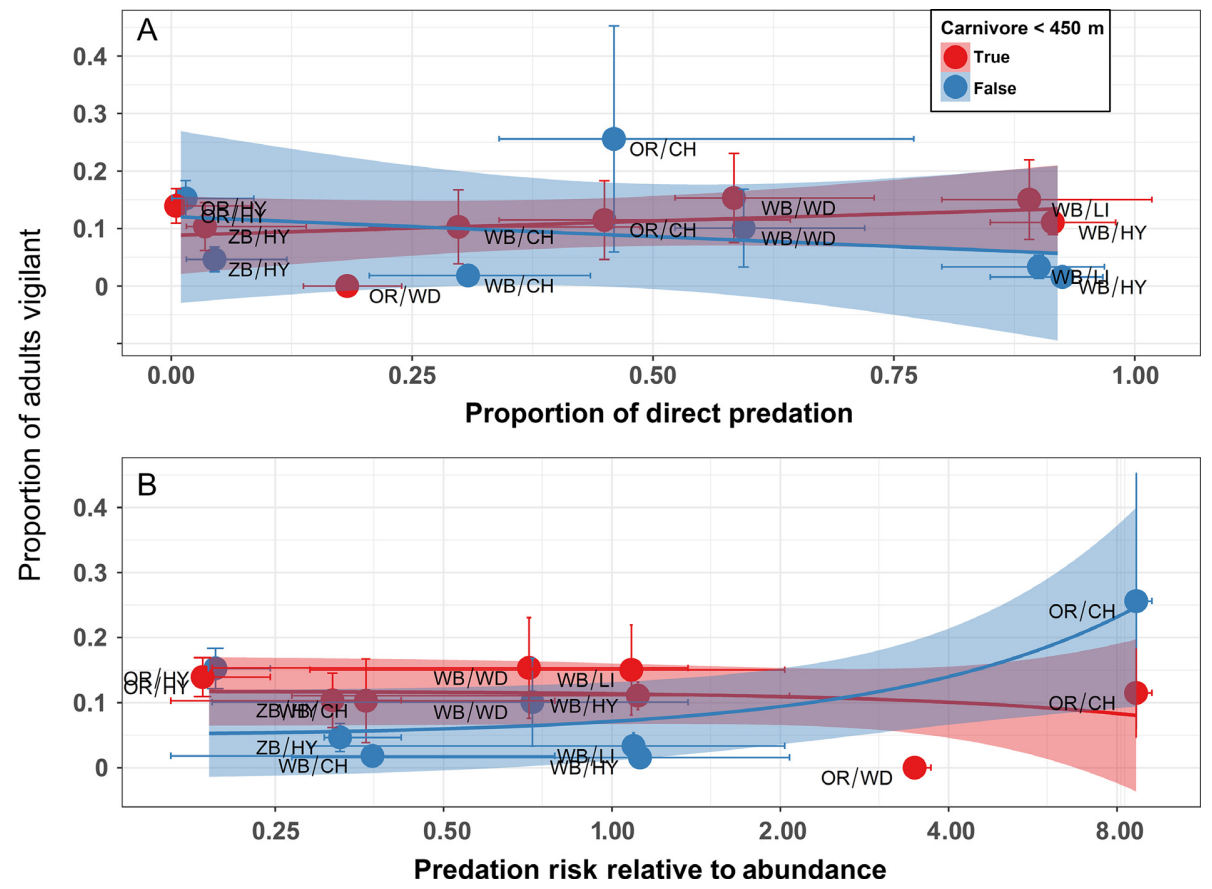

FIG. 7. (A) The relationship of vigilance by each prey species to the proportion of kills it constituted for each predator species, for observations with predators closer (red) or farther (blue) than $450 \mathrm{~m}$. (B) The relationship of vigilance by each prey species to the per-capita risk posed by each predator species, for observations with predators closer (red) or farther (blue) than $450 \mathrm{~m}$. Abscissa is log-scaled in panel B. Whiskers show binomial 95\% confidence limits. Point labels identify prey (OR, oribi; WB, wildebeest; $\mathrm{ZB}$, zebra) and then predator (CH, cheetah; HY, spotted hyena; LI, lion; WD, African wild dog). [Color figure can be viewed at wileyonlinelibrary.com] 
Karongwe Game Reserve found that "avoidance for the space use of predators generally did not correspond to the relative predation threat from those predators" (Thaker et al. 2011). Similarly, research in the OlkiramatianShompole region at edge of the Greater Serengeti Ecosystem found that the strength of behavioral responses by five ungulates did not correlate with patterns of predation on those species by lions and hyenas (Creel et al. 2014). In Liuwa, despite substantial variation in patterns of selection of each ungulate species by each predator species (Fig. 4), there was no detectable correlation between measures of direct predation and the strength of antipredator responses (Fig. 7). Thus, studies of ungulates and large carnivores to date (though still few) have consistently found that antipredator responses vary, but not in a manner that correlates with levels of direct predation.

This lack of correlation complicates efforts to understand the total limiting effect of predation on ungulates. Conceptual models (Creel and Christianson 2008) and empirical results like those reported here both suggest that we cannot simply assume that strong direct predation will be associated with strong risk effects. Consequently, we cannot assume that variation among prey species in direct predation provides an unambiguous measure of variation in the total limiting effect of predation. For example, species such as topi (Damaliscus lunatus topi) and waterbuck (Kobus ellipsiprymnus) typically experience little direct predation, although their size and morphological defenses are strikingly similar to sympatric species that experience strong predation. We must consider the possibility that such patterns are due to variation in the balance between direct predation and risk effects, rather than variation in the limiting effect of predation. Rather than correlating with direct predation risk itself, the strength of antipredator response should correlate with the reduction in direct predation risk that it yields.

When considering the lack of a detectable relationship between behavioral responses and patterns of direct predation, it is important to recall that the data revealed strong behavioral responses to predator presence (Fig. 2), and there was substantial variation in direct predation among predator-prey pairs (Fig. 4). Predation risk varied, and behavioral responses to predators varied, but the two patterns were uncorrelated.

Animals can only respond to the risks that they perceive, and one might argue that perceived risk should be a better predictor of vigilance than risk itself. However, responses to perceived risk will only be favored by natural selection if perceived risk accurately reflects the true risk of predation. Risk itself (and not the animal's perception) is the selection pressure shaping antipredator responses. Alternatively, one might argue that in predator-rich communities, it may be optimal to respond strongly to all predators, rather than running the risk of failing to respond when needed. However, this explanation tends to run contrary to the observation that vigilance varied appreciably among predatorprey pairs (Fig. 7), just not in a manner that correlated with patterns of direct predation.
Because the proportion of time spent foraging and vigilant summed to less than two-thirds of the time budget, it was not logically necessary that feeding would decline with increased vigilance, but this trade-off was nonetheless strong for the ungulate community in Liuwa, as with other studies of African ungulates (Crosmary et al. 2012, Periquet et al. 2012, Barnier et al. 2014, Creel et al. 2014). While there was no detectable relationship between antipredator responses and the threat posed by each carnivore, our results suggest that the effect of predation risk on foraging might be particularly strong for oribi, whose levels of proactive vigilance (when predators were not nearby [Broekhuis et al. 2013]) were substantially higher than those of wildebeest or zebra, and were similar to their own level of reactive vigilance when a carnivore was within $450 \mathrm{~m}$. In most systems, predators will typically be absent from the immediate vicinity of prey more often than they are present (though see Creel et al. 2013). Consequently, variation in the level of proactive vigilance is likely to be an important driver of the manner in which prey behavior partitions the total cost of predation into direct mortality and risk effects. Consistent with this interpretation, wildebeest experienced the heaviest direct predation, but also showed significantly lower proactive vigilance than the other species.

The most widely supported hypothesis for the evolution of inducible defenses is that conditional expression reduces the costs of defense: if defenses do not carry costs, their expression is not expected to depend on risk (Relyea and Werner 1999, Tollrian and Harvell 1999, Relyea and Auld 2004). Despite this general pattern, there has been debate over the costs of increased antipredator defenses in ungulates. Ungulates commonly respond to increased predation risk with increased vigilance, altered patterns of aggregation, reduced foraging, retreat to safe habitats, and altered diets (Prins and Iason 1989, Hunter and Skinner 1998, Caro 2005, Creel et al. 2005, 2014, Valeix et al. 2009, Christianson and Creel 2010, Periquet et al. 2012, Barnier et al. 2014). In some ungulates, these responses are associated with reduced reproduction (Creel et al. 2007, 2011, Christianson and Creel 2014, Cherry et al. 2016) as in other taxa (Peckarsky et al. 1993, Boonstra et al. 1998, Zanette 2003, Pangle et al. 2007, Zanette et al. 2011). Some authors nonetheless suggest that ungulates can respond to predation risk with no measurable demographic cost, essentially arguing that predation carries no costs beyond direct mortality (Middleton et al. 2013, White et al. 2011). Particularly in field studies, problems of methodology can easily mask the costs of antipredator responses (Creel et al. 2011, 2013) or allow them to be mistaken for bottom-up effects (Creel and Christianson 2008, Christianson and Creel 2014). Moreover, a cost that can be compensated for remains a cost, if it reduces the scope to address other constraints on foraging success.

The data provide a direct test for differences in the responses of prey to stalkers and coursers, and did not support the hypothesis that hunting mode is a reliable 
predictor of the magnitude of response. Despite including all members of the carnivore guild and examining responses by all of their common prey, the power of these tests is somewhat constrained by the number of species pairs (15) that could be considered. It also remains possible that responses to long-term variation in risk posed by stalkers and coursers ("risky places," sensu Creel et al. [2008]) differ from responses to the shortterm variation in risk examined here ("risky times," sensu Creel et al. [2008]).

Finally, our understanding of the role of risk effects in prey dynamics continues to be impeded by two problems. First is the problem discussed by Lank and Ydenberg (2003), that measurements of direct predation remain rare, but even these are not fundamentally a measure of the risk that prey would experience if they did not take action to reduce predation. Second is the problem that predation and the costs of avoiding it are measured in different currencies, and relating these measures to fitness or population dynamics remains difficult. Conceptually, the optimal level of antipredator response could be determined from a plot relating fitness costs (of reduced foraging, etc.) and fitness benefits (of reduced direct predation) to the strength of response, and determining where the benefits most greatly exceed the costs. Logically, the benefits of vigilance are likely to show an asymptote (once scanning rates are high enough to virtually guarantee predator detection), while costs are likely to continue increasing in a linear or even accelerating manner. We note that mean vigilance in the immediate presence of large carnivores was about $12-13 \%$ for all the prey species in this study, suggesting that this might be a general optimum.

In summary, the relationship between direct predation and the strength of antipredator responses is rarely measured. As in prior work on the responses of ungulates to risk (Thaker et al. 2011, Creel et al. 2014) and experimental studies with other taxa (Relyea 2001b, Relyea and Auld 2004), data from large carnivores and their ungulate prey in LPNP reveal no relationship. This lack of correlation implies that if antipredator responses carry costs, variation in direct predation does not accurately describe variation in the total limiting effect of predation.

\section{ACKNOWLEDGMENTS}

We thank the Zambia Department of National Parks and Wildlife for permission to work in LPNP, and the Worldwide Fund for Nature-Netherlands, National Science Foundation Animal Behavior Program (IOS-1145749), Painted Dog Conservation Inc., National Geographic's Big Cats Initiative and the African Parks Network for support of the research. Our sincere thanks to Teddy Mukula, Kings Chimungu, Stanley Mwanza, Finn Corry, Vera Hoffman, Graeme Ellis, and Shadrach Mwaba of the ZCP Liuwa Team for their dedicated work in the field.

\section{Literature Cited}

Balme, G., L. Hunter, and R. Slotow. 2007. Feeding habitat selection by hunting leopards Panthera pardus in a woodland savanna: prey catchability versus abundance. Animal Behaviour 74:589-598.

Barnier, F., M. Valeix, P. Duncan, S. Chamaillé-Jammes, P. Barre, A. J. Loveridge, D. W. Macdonald, and H. Fritz. 2014. Diet quality in a wild grazer declines under the threat of an ambush predator. Proceedings of the Royal Society B 281:20140446.

Blanchard, P., and H. Fritz. 2007. Induced or routine vigilance while foraging. Oikos 116:1603-1608.

Boinski, S., L. Kauffman, A. Westoll, C. M. Stickler, S. Cropp, and E. Ehmke. 2003. Are vigilance, risk from avian predators and group size consequences of habitat structure? A comparison of three species of squirrel monkey (Saimiri oerstedii, S. boliviensis, and S. sciureus). Behaviour 140:14211467.

Boonstra, R., D. Hik, G. R. Singleton, and A. Tinnikov. 1998. The impact of predator-induced stress on the snowshoe hare cycle. Ecological Monographs 68:371-394.

Broekhuis, F., G. Cozzi, M. Valeix, J. W. McNutt, and D. W. Macdonald. 2013. Risk avoidance in sympatric large carnivores: reactive or predictive? Journal of Animal Ecology 82: $1098-1105$.

Caro, T. 2005. Antipredator defenses in birds and mammals. University of Chicago Press, Chicago, Illinois, USA.

Chandler, R. 2014. Distance sampling analysis in unmarked. Comprehensive R Archive Network, Vienna, Austria.

Cherry, M. J., K. E. Morgan, B. T. Rutledge, L. M. Conner, and R. J. Warren. 2016. Can coyote predation risk induce reproduction suppression in white-tailed deer? Ecosphere 7: e01481.

Christianson, D., and S. Creel. 2010. A nutritionally mediated risk effect of wolves on elk. Ecology 91:1184-1191.

Christianson, D., and S. Creel. 2014. Ecosystem scale declines in elk recruitment and population growth with wolf colonization: A before-after-control-impact approach. PLoS ONE 9: e102330.

Clinchy, M., L. Zanette, R. Boonstra, J. C. Wingfield, and J. N. M. Smith. 2004. Balancing food and predator pressure induces chronic stress in songbirds. Proceedings: Biological Sciences 271:2473-2479.

Courbin, N., A. J. Loveridge, D. W. Macdonald, H. Fritz, M. Valeix, E. T. Makuwe, and S. Chamaillé-Jammes. 2015. Reactive responses of zebras to lion encounters shape their predator-prey space game at large scale. Oikos 125 : 829-983.

Creel, S., and D. Christianson. 2008. Relationships between direct predation and risk effects. Trends in Ecology and Evolution 23:194-201.

Creel, S., J. Winnie, B. Maxwell, K. L. Hamlin, and M. Creel. 2005. Elk alter habitat selection as an antipredator response to wolves. Ecology 86:3387-3397.

Creel, S., and N. M. Creel. 2002. The African wild dog: behavior, ecology and conservation. Princeton University Press, Princeton, New Jersey, USA.

Creel, S., D. Christianson, S. Liley, and J. A. Winnie. 2007. Predation risk affects reproductive physiology and demography of elk. Science 315:960.

Creel, S., J. Winnie Jr, D. Christianson, and S. Liley. 2008. Time and space in general models of antipredator response: tests with wolves and elk. Animal Behaviour 76:1139-1146.

Creel, S., D. Christianson, and J. Winnie Jr. 2011. A survey of the effects of wolf predation risk on pregnancy rates and calf recruitment in elk. Ecological Applications 21:2847-2853.

Creel, S., J. A. Winnie, and D. Christianson. 2013. Underestimating the frequency, strength and cost of antipredator responses with data from GPS collars: an example with wolves and elk. Ecology and Evolution 3:5189-5200. 
Creel, S., P. Schuette, and D. Christianson. 2014. Effects of predation risk on group size, vigilance, and foraging behavior in an African ungulate community. Behavioral Ecology 25: 773-784.

Cresswell, W., and J. L. Quinn. 2010. Attack frequency, attack success and choice of prey group size for two predators with contrasting hunting strategies. Animal Behaviour 80:643-648.

Cresswell, W., and J. L. Quinn. 2013. Contrasting risks from different predators change the overall nonlethal effects of predation risk. Behavioral Ecology 24:871-876.

Cresswell, W., J. Lind, and J. L. Quinn. 2010. Predator hunting success and prey vulnerability: quantifying the spatial scale over which lethal and non-lethal effects of predation occur. Journal of Animal Ecology 79:556-562.

Crosmary, W. G., M. Valeix, H. Fritz, H. Madzikanda, and S. D. Côté. 2012. African ungulates and their drinking problems: hunting and predation risks constrain access to water. Animal Behaviour 83:145-153.

Droge, E., S. Creel, J. M'soka, and M. Becker. 2017. Spatial and temporal avoidance of risk within a large carnivore guild. Ecology and Evolution 7:189-199.

Fortin, D., M. S. Boyce, and E. H. Merrill. 2004a. Multi-tasking by mammalian herbivores: overlapping processes during foraging. Ecology 85:2312-2322.

Fortin, D., M. S. Boyce, E. H. Merrill, and J. M. Fryxell. $2004 b$. Foraging costs of vigilance in large mammalian herbivores. Oikos 107:172-180.

Hopcraft, J. G., A. R. E. Sinclair, and C. Packer. 2005. Planning for success: Serengeti lions seek prey accessibility rather than abundance. Journal of Animal Ecology 74:559-566.

Hunter, L. T. B., and J. D. Skinner. 1998. Vigilance behaviour in African ungulates: the role of predation pressure. Behaviour 135:195-211.

Kauffman, M. J., N. Varley, D. W. Smith, D. R. Stahler, D. R. MacNulty, and M. S. Boyce. 2007. Landscape heterogeneity shapes predation in a newly restored predator prey system. Ecology Letters 10:690-700.

Kerfoot, W. C., and A. Sih. 1987. Predation: direct and indirect impacts on aquatic communities. University Press of New England, Hanover, New Hampshire, USA.

LaManna, J. A., and T. E. Martin. 2016. Costs of fear: behavioural and life-history responses to risk and their demographic consequences vary across species. Ecology Letters 19:403-413.

Lank, D. B., and R. C. Ydenberg. 2003. Death and danger at migratory stopovers: problems with "predation risk." Journal of Avian Biology 34:225-228.

Liley, S., and S. Creel. 2008. What best explains vigilance in elk: characteristics of prey, predators, or the environment? Behavioral Ecology 19:245-254.

Lima, S. L. 1998. Nonlethal effects in the ecology of predatorprey interactions. BioScience 48:25-34.

Lima, S. L. 2002. Putting predators back into behavioral predator-prey interactions. Trends in Ecology and Evolution 17:70-75.

Lima, S. L., and L. M. Dill. 1990. Behavioral decisions made under the risk of predation: a review and prospectus. Canadian Journal of Zoology 68:619-640.

Lind, J., and W. Cresswell. 2005. Determining the fitness consequences of antipredation behavior. Behavioral Ecology 16:945-956.

Middleton, A. D., M. J. Kauffman, D. E. McWhirter, M. D. Jimenez, R. C. Cook, J. G. Cook, S. E. Albeke, H. Sawyer, and P. J. White. 2013. Linking antipredator behaviour to prey demography reveals limited risk effects of an actively hunting large carnivore. Ecology Letters 16:1023-1030.
M'soka, J., S. Creel, M. S. Becker, and E. Droge. 2016. Spotted hyaena survival and density in a lion depleted ecosystem: The effects of prey availability, humans and competition between large carnivores in African savannahs. Biological Conservation 201:348-355.

M'soka, J., S. Creel, M. S. Becker, and J. Murdoch. 2017. Ecological and anthropogenic effects on the density of migratory and resident ungulates in a human-inhabited area. African Journal of Ecology, https://doi.org/10.1111/aje.12398, in press.

Pangle, K. L., S. D. Peacor, and O. E. Johannsson. 2007. Large nonlethal effects of an invasive invertebrate predator on zooplankton population growth rate. Ecology 88:402-412.

Peacor, S. D., and E. E. Werner. 2001. The contribution of trait-mediated indirect effects to the net effects of a predator. Proceedings of the National Academy of Sciences USA 98:3904-3908

Peckarsky, B. L. 1996. Alternative predator avoidance syndromes of stream-dwelling mayfly larvae. Ecology 77:1888-1905.

Peckarsky, B. L., and A. R. McIntosh. 1998. Fitness and community consequences of avoiding multiple predators. Oecologia 113:565-576.

Peckarsky, B. L., C. A. Cowan, M. A. Penton, and C. Anderson. 1993. Sublethal consequences of stream-dwelling predatory stoneflies on mayfly growth and fecundity. Ecology 74: 1836-1846.

Peckarsky, B. L., P. A. Abrams, D. I. Bolnick, L. M. Dill, J. H. Grabowski, B. Luttbeg, J. L. Orrock, S. D. Peacor, E. L. Preisser, and O. J. Schmitz. 2008. Revisiting the classics: considering nonconsumptive effects in textbook examples of predator-prey interactions. Ecology 89:2416-2425.

Periquet, S., et al. 2012. Influence of immediate predation risk by lions on the vigilance of prey of different body size. Behavioral Ecology 23:970-976.

Preisser, E. L., D. I. Bolnick, and D. T. Blumstein. 2005. Scared to death? The effects of intimidation and consumption in predator prey interactions. Ecology 86:501-509.

Prins, H. H. T., and G. R. Iason. 1989. Dangerous lions and nonchalant buffalo. Behaviour 108:262-296.

R Development Core Team 2008. R: A language and environment for statistical computing. R Foundation for Statistical Computing, Vienna, Austria. www.r-project.org

Relyea, R. A. 2001a. Morphological and behavioral plasticity of larval anurans in response to different predators. Ecology 82:523-540.

Relyea, R. A. 2001b. The relationship between predation risk and antipredator responses in larval anurans. Ecology 82: 541-554.

Relyea, R. A., and J. R. Auld. 2004. Having the guts to compete: How intestinal plasticity explains costs of inducible defences. Ecology Letters 7:869-875.

Relyea, R. A., and E. E. Werner. 1999. Quantifying the relationship between predator-induced behavior and growth performance in larval anurans. Ecology 80:2117-2124.

Schmitz, O. J. 2008. Effects of predator hunting mode on grassland ecosystem function. Science 319:952-954.

Schmitz, O. J., A. P. Beckerman, and K. M. O’Brien. 1997. Behaviorally mediated trophic cascades: effects of predation risk on food web interactions. Ecology 78:1388-1399.

Schmitz, O. J., V. Krivan, and O. Ovadia. 2004. Trophic cascades: the primacy of trait-mediated indirect interactions. Ecology Letters 7:153-163.

Skelly, D. K. 1992. Field evidence for a cost of behavioral antipredator response in a larval amphibian. Ecology 73: 704-708

Thaker, M., A. T. Vanak, C. R. Owen, M. B. Ogden, S. M. Niemann, and R. Slotow. 2011. Minimizing predation risk in 
a landscape of multiple predators: effects on the spatial distribution of African ungulates. Ecology 92:398-407.

Tollrian, R., and C. D. Harvell. 1999. The evolution of inducible defenses: current ideas. Pages 306-321 in R. Tollrian and C. D. Harvell, editors. The ecology and evolution of inducible defenses. Princeton University Press, Princeton, New Jersey, USA.

Valeix, M., A. J. Loveridge, S. Chamaillé-Jammes, Z. Davidson, F. Murindagomo, H. Fritz, and D. W. Macdonald. 2009. Behavioral adjustments of African herbivores to predation risk by lions: spatiotemporal variations influence habitat use. Ecology 90:23-30.

Werner, E. E., and S. D. Peacor. 2003. A review of traitmediated indirect interactions in ecological communities. Ecology 84:1083-1100.

Werner, E. E., J. F. Gilliam, D. J. Hall, and G. G. Mittelbach. 1983. An experimental test of the effects of predation risk on habitat use in fish. Ecology 64:1540-1548.
White, P. J., R. A. Garrott, K. L. Hamlin, R. C. Cook, J. G. Cook, and J. A. Cunningham. 2011. Body condition and pregnancy in northern Yellowstone elk: Evidence for predation risk effects?. Ecological Applications 21:3-8.

Winnie, J., and S. Creel. 2007. Sex-specific behavioural responses of elk to spatial and temporal variation in the threat of wolf predation. Animal Behaviour 73: 215-225.

Zanette, L.. 2003. Synergistic effects of food and predators on annual reproductive success in song sparrows. Proceedings: Biological Sciences 270:799-803.

Zanette, L. Y., A. F. White, M. C. Allen, and M. Clinchy. 2011. Perceived predation risk reduces the number of offspring songbirds produce per year. Science 334:1398-1401.

Zuur, A. F., E. N. Ieno, N. J. Walker, A. A. Savaliev, and G. M. Smith. 2009. Mixed effects models and extensions in ecology with R. Springer, New York, New York, USA. 